\title{
Blockchain For Education Purpose: Essential Topology
}

\author{
Qurotul Aini ${ }^{1}$, Ninda Lutfiani ${ }^{2}$, Nuke Puji Lestari Santoso ${ }^{3}$, \\ Sulistiawati ${ }^{4}$, Erna Astriyani ${ }^{5}$ \\ 1 Universiti Technology of Malaysia, ${ }^{2,3,4,5}$ University of Raharja \\ e-mail: qurotul@graduate.utm.my ${ }^{1}$, ninda@raharja.info ${ }^{2}$, nuke@raharja.info ${ }^{3}$, \\ sulsitiawati.wati@raharja.info ${ }^{4}$, erna.astriyani@raharja.info ${ }^{5}$
}

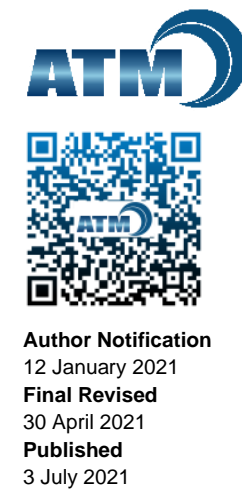

To cite this document :

Aini, Q., Lutfiani, N., Santoso, N., Sulistiawati, S., \& Astriyani, E. (2021). Blockchain For Education Purpose: Essential Topology. Aptisi Transactions on Management (ATM), 5(2), 112-120.

DOI :

https://doi.org/10.33050/atm.v5i2.1506

\begin{abstract}
Blockchain technology is known for its large-scale digitisation trends that play an important role in various organisations. Because it is distributed and decentralized from blockchain, this feature makes the role of blockchain technologies based on products and services significantly different from previous technology offerings. The value proposition offered by this blockchain is very suitable to be applied in education. After a few years this caused disruption from various industry circles, current educational areas including those that are very perceived by the Indonesian people there is no progress and road in place. It is possible that in the next ten years, the educational actors who are less sensitive will one by one fall because of the storm-cast disruption technology. The educational institution is one of which is aware of the advantages and extraordinary potential of blockchain technology. Among other things, a digital signature certificate is important in the context of digital archiving to verify the authenticity of a document in an educational institution. But the lack of education from the community makes ignorance about the point of the advantage of blockchain technology that can be applied in the field of education. Therefore, a public understanding of the blockchain is packaged in the form of ontology on the education shutter. This research in particular is exploring the ontology approach of using root merkle methods in providing solutions to the application of blockchain, including slow process and small storage space. This issue causes it to be very difficult for educational institutions to store processes and stages of learning into the blockchain. This research can be the cornerstone of identifying solutions from certificates of digital signatures in terms of archiving, authentication and digital verification on the design of a new breakthrough framework with an ontology approach to blockchain educational design.
\end{abstract}

Keywords: Blockchain, education, Markle root, ontology.

\section{Introduction}

Blockchain is known as an extraordinary development for recording and putting away information of an appropriated record with powerful security components. Blockchain innovation gives another way to deal with building trust in a conniving climate and in this way information uprightness, accessibility, detectability, and information the board security [1]. Blockchain innovation can improve the different characteristics in the framework in different angles, particularly in the instructive perspective, researchers consider that the fundamental benefits of blockchain innovation are straightforwardness, decentralization of organizations, confided in chains with genuine and irreversible information [2]. In any case, individuals' absence of information about blockchain innovation has become an obliviousness of the benefits that can be acquired in blockchain innovation. The utilization of innovation in the time of the advanced unrest has made innovation the primary need, science and innovation are growing quick to such an 
extent that innovation in this time is utilized in different fields of science. In this Revolution 5.0, individuals ought to be more instructed about further developed advances, as well as giving comfort regardless, just as to upset the improvement of the occasions [3].

The potential in education aspects of blockchain technology is rapidly growing, with a certificate in a digital signature for digital archiving to verify the authenticity of a document. Certificates are an important asset for educational institutions, which is a form of competitive advantage and to support careers, data on the blockchain is protected by several layers of secondary technology such as hashes, hashe chains, private-public keys, and P2P data distribution [4] . The presence of blockchain technology will no longer occur in document forgery or manipulation of user data. Technology that is increasingly sophisticated and growing has become an ease for the community, technology opportunities must be utilized well by educational institutions [5].

Blockchain structures containing headers (hashes and Merkle root) and transactions, proof of work (called miners in cryptocurrencies) are other features of the blockchain, represented as a secure platform for transferring data. Simply explain, it solves complex mathematical puzzles, which require great computational power, and every transaction or block will not apply unless it solves this puzzle [6]. The solving process includes validating the creator of the transaction, enough funds to transfer, avoiding double spend etc. Any node that can solve the puzzle, is eligible to create a new block that includes all verified transactions. After that, the block broadcasts to the network and all the other nodes record it in their ledbook. This paper means to make hypothetical commitments and perspectives to blockchains utilizing an ontological methodology [7]. Cosmology helps the way toward breaking down, separating, and incorporating data on the web, making it simple to make solid fundamental information, philosophy has been perceived as a helpful instrument for lessening theoretical vagueness and irregularities when distinguishing values in a specific space. Philosophy turned into a basic instrument for lessening intricacy by organizing spaces of interest in training for courses offered on authentications in advanced marks for computerized documenting. The production of metaphysics can obviously be considered as the initial move towards semantic training [8].

\section{Literature Review}

Philosophy is a portrayal (like the proper detail of a program) of ideas and connections that can exist for a specialist or local area of specialists. This definition is steady with the utilization of philosophy as a bunch of-idea definition, however more by and large. Also, that is unmistakably an alternate feeling of the word than its utilization in way of thinking. Metaphysics is frequently compared to an order of class scientific classification, however the meaning of class, and the relationship of subsumption, yet philosophy need not be restricted to these structures [9]. Metaphysics is additionally not restricted to moderate definitions, that is, definitions in the conventional rationale sense that just present wording and don't add information on the world. Designing cosmology alongside semantic web innovation, empowering semantic turn of events and operational stream demonstrating needed for blockchain plan [10]. Blockchain configuration depends on the essential standards of Internet design: endurance (Internet correspondence should proceed notwithstanding organize or Gateway misfortune), various sorts of administrations (numerous kinds of correspondence administrations), different organizations (different sorts of organizations), circulated asset the executives, productivity, simplicity of facilitating, and obligation regarding asset use [11]. Blockchain metaphysics created by de Kruijff and WEIGAND has now evolved in the field of instruction, notwithstanding, different viewpoints that apply blockchain cosmology are business angles to improve the nature of substance from a bookkeeping point of view just as framework quality as far as evaluating and interoperability. This is appeared to recognize the Datalogic level, an Infological and an Essential (reasonable) level. Copyright the executives is adjusted to copyright cosmology used to construct beneficial applications, rich data displaying and thinking alongside the capacity of perpetual and responsible data that gives certainty and trust in demonstrated rights explanations [12].

The idea of modern blockchain as the utilization of blockchain innovation in the business with productive Integration of IoT, M2M, and agreement calculations. It gives open yet secure data stockpiling and stage trade for different partners to accomplish receptiveness, interoperability and decentralization in the business 4.0 time [13]. Distributed organizations are made to improve the productivity of information dividing among participators. The basic structure of blockchains was introduced, including blocks, chains, and transactions. Blockchains are used for data storage 
through cryptography which ensures only eligible participators can access the appropriate data. Sensitive change characteristics of the blockchain can ensure the authenticity of the data as well. In addition, the Smart Contract concept can be used to perform multiple actions automatically to improve data sharing efficiency [14].

\section{Research Methods}

In a square, have each exchange as all hashes and become an outcome that is merkle root. A progression of hashes are associated through a header, and with a header that has a reference to the past hash duplicate structure the capacity to chain together exchanges. Duplicates of every exchange and hash are then matched, combined, matched once more, and hashed again until one fixed hash is merkle root. In this examination utilizing merkle root method [15]. Merkle root is important for the header of a square, for example the hash of the multitude of hashes of exchanges inside the square, each square additionally stores a hash of the past block header, tying the squares together [16]. This guarantees that the exchange can't be adjusted without altering the square that records it, and every one of the accompanying squares, as such permit to securely confirm that the exchange has been gotten in the organize and download just the little square headers and merkle tree downloads in the whole square chain that are not significant. Square headers should incorporate legitimate Merkle roots got from all exchanges in the square [17].

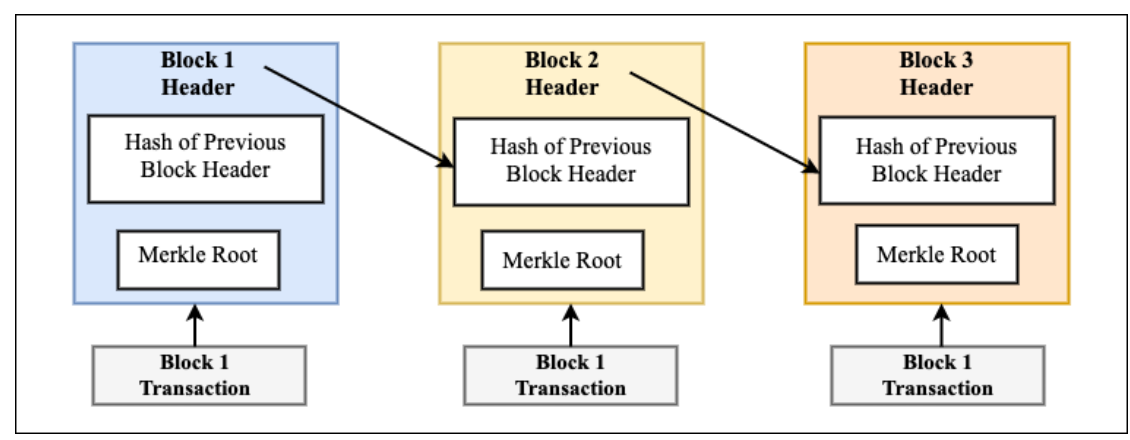

Figure 1. Simplified Blockchain Concepts.

Figure 1 above shows a worked on rendition of the blockchain. Squares of at least one new exchanges are gathered into the exchange information part of a square. A progression of hashes are associated through the header, and with a header that has a reference to the past hash duplicate structure the capacity to chain together exchanges [18]. Duplicates of every exchange and hash are then matched, combined once more, and hashed again until one hash remains. Blockchains are block groupings, which hold a total rundown of exchange records like customary public Ledgers. Figure 1 represents an illustration of blockchain. With past hash blocks contained in block headers, blocks have just one parent block [19]. It ought to be noticed that hash squares will likewise be put away in the ethereum blockchain. The principal square of the blockchain is known as the Genesis Block which has no parent block. We at that point clarify the inside blockchain in detail [20].

Merkle root is put away in the header block. Square headers are important for bitcoin blocks that get hashes in the diggers cycle. It contains the hash of the last square, a Nonce, and the root hash of all exchanges in the current square in the Merkle tree. So having Merkle root in the header block makes the exchange a Proof [21]. As this root hash remembers hashes of all exchanges for blocks, these exchanges can bring about circle space stockpiling. The body block comprises of counter exchanges and exchanges. The most extreme number of exchanges that can contain blocks relies upon the square size and size of every exchange. Blockchains utilize topsy-turvy cryptographic components to approve exchange validation. Advanced marks dependent on balanced cryptography are utilized in dishonest conditions. We next momentarily portrayed the computerized signature [22].

Merkle root is put away in the header block. Each square additionally stores a hash of the past block header, affixing the squares together. This guarantees exchanges can't be adjusted without altering the recorded squares and every one of the accompanying squares. Exchanges are likewise tied together [23]. Bitcoin wallet programming gives the impression of being shipped 
off and from the wallet, purchase bitcoins are truly moving from one exchange to another. Each past spending exchange is gotten in at least one past exchanges, so the contribution of one exchange is the yield of the past exchange. A solitary exchange can make numerous yields, as happens when shipping off different locations, however any yield from a given exchange must be utilized as an information once in a square chain. Each ensuing reference is a twofold spend on an endeavor to spend twice [24].

\begin{tabular}{|c|}
\hline Block Version \\
\hline $\begin{array}{c}\text { Previous Block } \\
\text { Hash }\end{array}$ \\
\hline $\begin{array}{c}\text { Merkle Tree } \\
\text { Root }\end{array}$ \\
\hline Timestamp \\
\hline Nonce \\
\hline nBits \\
\hline
\end{tabular}

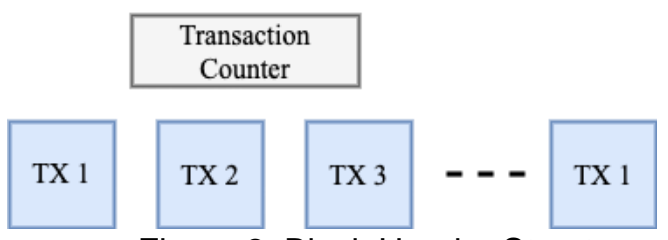

Figure 2. Block Header Structure.

In figure 2 shown the square construction, each square comprises of a few renditions of the square, the parent block hash, the Merkle tree root hash, timestamp, nBits, and Nonce [23]. The square form represents the approval rules to follow. The parent hash block addresses the hash of the past square to frame a chain. 256-digit hash [25]. Timestamp addresses the time in short order, while nBits show the hashing of the objective, which addresses the square limit to be substantial. nBits is a whole number that hash the header should be beneath or equivalent to that of a header that gets substantial from the blockchain. A Nonce is an irregular 4-byte number created to deliver hashes that make the square legitimate. The hash block begins with nothing and the number builds zero in rapidly so it's difficult to sort out the hash. In this manner, diggers consistently ascertain and surmise the nonce that will deliver the specific hash (counting the quantity of zeros toward the start), which will make the square substantial, as in figure 2 above [26].

As such, excavators should deliver yields that meet certain necessities while embeddings Nonce into the hashing calculation. Diggers use hardness to figure the right worth calculation until the relating yield esteem is found. Such computations are vital in light of the fact that the info information creates a totally unique yield. Consequently, this computation should show the precise worth of the yield addressing the special information [27].

Merkle tree keeps up information uprightness. In the event that there is a solitary detail of the exchange or the request wherein the exchange changes, at that point this change is reflected in the hash of the exchange. This change will course up the Merkle tree to Merkle root, change the merkle root worth and consequently not approve the square. So everybody can see that the merkle tree considers a speedy and straightforward test whether a specific exchange is remembered for the set or not [28]. 


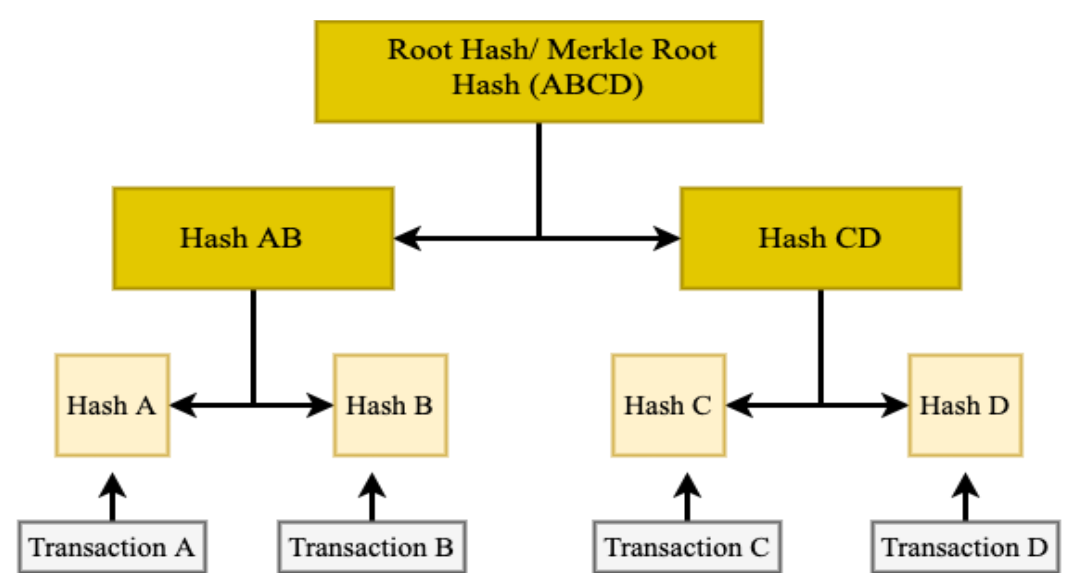

Figure 3. Blockchain Merkle tree Root.

The hash of all exchanges in the square is called Merkle root as demonstrated in figure 3. Each pair of exchange hashes is consolidated together until a solitary hash is gone after all exchanges, for example called Root hash or Merkle root. For instance, hashes of exchange An and exchange $B$ are consolidated together to create another hash called an $A B$ hash; a similar cycle is done with exchanges $C$ and $D$; Finally, the root hash (ABCD hash) is produced by joining the $A B$ hash and $C D$ hash. Exchanges and related counters are situated in the square segment. The square size and exchange size are liable for deciding the quantity of exchanges in a solitary square [29].

To approve exchange verification, blockchains utilize symmetric/lopsided cryptographic instruments, where private keys are utilized to sign and scramble information on the sender's side. Public keys are utilized to decode information on the collector side. The exchange marking measure brings about what is known as a computerized signature. Computerized marks include two stages, to be specific: Signing and check [30]. For instance, when client $X$ makes an exchange for client $Y$, it returns a particular exchange hash esteem. The encryption cycle is performed utilizing the client's private key $X$ (sender). Unique information and scrambled hashes are shipped off $Y$. clients on the organization can unscramble hashes utilizing the client's public $X$ key. In this way, client $Y$ decodes the got hash and contrasts it and hashes got from the information gotten utilizing the client $X$ hash capacity to confirm the exchange [31].

At the point when an exchange is added to a square, the exchange and any remaining exchanges in the square just as the hash of the past block make the square hash. This makes a square chain that each alludes to the past block and no information in this square or information in the exchange can be changed without changing the hash of the exchange and accordingly the hash of the square and henceforth the hash of every single resulting block [32]. Merkle root is the last hash, after hashing on each transaction in one block. To store storage, some blocks may not store all transactions, but only store intermediate hashes. Reducing storage by skipping transactions like this will reduce overall system security. Because this lightweight node cannot verify the transaction and is therefore vulnerable to attack. If transactions in the block are changed, merkle root must be updated. If the Merkle root block is updated, the block data will be updated. Therefore the block must be re-hashed [33].

\section{Results and Discussion}

For every data framework, there are 3 necessities that should be met to guarantee security, specifically: privacy, trustworthiness, and accessibility. Since blockchain is decentralized, it can ensure the usefulness of a worldwide framework regardless of whether at least one hubs. In blockchain, classification incorporates getting a client's private key as it is needed alongside a public key for the framework or imitating another person (taking identity) [34]. Public and private keys are utilized to guarantee trustworthiness and security while trading data. 
There is an interesting private key for the client, which ensures responsibility for data for a specific client. The client signs the data with their private key to exhibit his power all through the organization. A public key comes from a private key that depends on a particular calculation that utilizes the framework. Public keys are disseminated across the organization since they are irreversible (private keys can't emerge out of open keys). Different clients should utilize a public key to get to the data [35].

Since each square is associated with the past block by entering the past block hash. This hash incorporates Merkle's root hash of all exchanges in the past block. In the event that one exchange were to transform, it would change the Merkle hash tree, yet so would the hash contained in the square change. Likewise, each ensuing square should be refreshed to mirror this change. On account of confirmation of work, the measure of energy needed to recalculate Nonce for this square and each resulting square will be a hindrance. Then again, in the event that somebody alters an exchange in a square without going through the fundamental strides to refresh the following square, it will be not difficult to recalculate the hash utilized in the impede and verify that something isn't right [36].

Blockchain security components forestall hacking through appropriated agreement, guaranteeing the security of incorporated information the executives and capacity frameworks as all exchanges are needed to be checked and approved by gatherings or networks of excavators [37]. Moreover, the blockchain network is checked by all hubs in the organization, and every programmer hub doesn't have the ability to embed controlled squares into the record, as all hubs hold a duplicate of the blockchain [38]. Consequently, in any event, hacking a few records won't influence the blockchain, since duplicates of the blockchain given by others are viewed as dependable reinforcements. Blockchain frameworks can get networks from certain noxious exercises. Nonetheless, some of them may make issues the blockchain network [39].

In certain executions, information units can be handled to deliver hashes. Cryptographic constructions, for example, Merkle root or other cryptographic designs, might be made dependent on hashes and distributed on block chains or conveyed records [40]. Proof identified with the information unit can be given. Proof can permit autonomous check that the information unit is confirmed. Receptive to acquiring information units of at any rate one information unit to be confirmed, information units can be wanted. Related proof can be gotten, where the proof acquired incorporates a record portraying the hash position among at least one different hashes in the distributed cryptographic design. Cryptographic constructions can be recreated dependent on the Evidence Index [41]. Information unit certificate can be confirmed by demonstrating that the hash has a place with the foundation of the distributed cryptographic design. Square binds can be utilized to verify the presence, uprightness, as well as responsibility for or interchanges [42]. The current divulgence depicts the acknowledgment of information unit majority, hashing a majority of information units to give a majority of hashes, singular hashes turning out to be extraordinary cryptographic identifiers of the suitable information units so singular hashes can identify with the fitting information units and individual hashes can't be utilized alone to acquire the proper information units. The aftereffect of the investigation was a merkle root strategy in blockchain cosmology way to deal with create computerized marks in chronicling check [43].

\section{Conclusion}

Data and correspondence innovation assumes a significant part in this day and age. Be that as it may, the part of instruction in the space dealt with numerous issues because of absence of training in learning innovation, powerlessness to interface various frameworks, and helpless interoperability between frameworks. As of late, consideration has developed towards the utilization of blockchain in schooling. Blockchain shows colossal potential in the instructive area as it addresses issues identified with advanced mark records by giving protection, security, interoperability, approval, and verification. Blockchain offers answers for genuine issues today, for example, less effective extra room, and shaky information. In this paper, a methodical survey of different agreement calculations identifying with instruction and existing work has been completed. This paper features their essential thoughts and difficulties. In light of an efficient audit, contextual investigations coordinating blockchain into the part of instruction are proposed to address research holes from existing positions.

All Merkle trees must be constructed in accordance with the unique Merkle tree construction originally implemented by Satoshi, which relies on the tree construction of the ordered list i.e., calculating the list from the original BLAKE-256 data (e.g. transaction id), then if 
the list is empty, return the 32-byte hash root of all zeroes; On the contrary, and the list contains two or more items, including: If the number of items left in the list is strange, duplicate the final hash. Combines each pair of adjacent entries with the BLAKE-256 hash of the two entries combined together. The list will have a ceil (N/2) entry remaining after merging adjacent entries, and returns the last remaining item in the list as Merkle root. Merkle Trees is an information structure that can take the ' $n$ ' number of hashes and address them with a solitary hash. In blockchain terms, merkle tree sums up all exchanges on the square by creating a solitary unique finger impression of the whole exchange set. It keeps up information respectability. On the off chance that one detail is in the exchange or the request where the exchange changes, so does Merkle root. Utilizing The Merkle tree considers a fast and straightforward trial of whether a specific exchange is remembered for the set or not. The single code generated by the Merkle tree is called Merkle root. Each block in the blockchain has exactly one. Merkle root is an important part of the data because it allows the computer to verify information with exceptional speed and efficiency.

This paper explores the idea of an ontology approach in utilizing the merkle root method to provide solutions to the application of blockchain technology, Blockchain can support the same data without losing user control in a decentralized environment. From this research, it can be concluded that with an approach in the form of ontology is able to contribute theoretically and views to blockchain, in addition to helping in the process of analyzing, extracting, and integrating information on the web, making it easier to create strong basic knowledge, ontology has been recognized as a useful instrument to reduce conceptual ambiguity and inconsistencies when identifying values in a particular domain. This research can also be a reference for identifying solutions from certificates encoded in digital hand Q\&amp;A for archiving, authentication and digital verification in the Design of new breakthrough frameworks in the ontology approach to blockchain technology design.

\section{Acknowledgment}

The author would like to thank the supervisors who have participated in helping to complete this research, the author especially appreciates the tremendous hard work in simplifying the algorithm, as it has helped to improve the presentation of these results.

\section{References}

[1] U. Rahardja, Q. Aini, M. Yusup, and A. Edliyanti, "Penerapan Teknologi Blockchain Sebagai Media Pengamanan Proses Transaksi E-Commerce," Comput. Eng. Sci. Syst. J., vol. 5, no. 1, pp. 28-32.

[2] M. Yeni and D. Kumala, "Teknologi Blockchain untuk Transparansi dan Keamanan pada Era Digital," 2020.

[3] M. Yusup, Q. Aini, D. Apriani, and P. Nursaputri, "PEMANFAATAN TEKNOLOGI BLOCKCHAIN PADA PROGRAM SERTIFIKASI DOSEN," in SENSITIf: Seminar Nasional Sistem Informasi dan Teknologi Informasi, 2019, pp. 365-371.

[4] Y.-C. Liang, "Blockchain for dynamic spectrum management," in Dynamic Spectrum Management, Springer, 2020, pp. 121-146.

[5] L. Ren and P. A. S. Ward, "Pooled mining is driving blockchains toward centralized systems," in 2019 38th International Symposium on Reliable Distributed Systems Workshops (SRDSW), 2019, pp. 43-48.

[6] K. Patel and M. L. Das, "Transcript management using blockchain enabled smart contracts," in International Conference on Distributed Computing and Internet Technology, 2020, pp. 392-407.

[7] J. Vlieghe and P. Zamojski, "Towards an immanent ontology of teaching Leonard Bernstein as a case-study," Ethics Educ., vol. 15, no. 1, pp. 1-17, 2020.

[8] O. Djama and Z. Boufaida, "Instantiation of the multi-viewpoints ontology from a resource," Int. J. Comput. Appl., pp. 1-12, 2020.

[9] E. Massaro, A. Athanassiadis, A. Psyllidis, and C. R. Binder, "Ontology-based integration of urban sustainability indicators," Sustain. Assessments Urban Syst., p. 332, 2020.

[10] N. Sfetcu, "Blockchain Design and Modelling," 2019.

[11] H. Weigand, I. Blums, and J. de Kruijff, "Shared ledger accounting-implementing the economic exchange pattern in DL technology," in International Conference on Advanced 
Information Systems Engineering, 2018, pp. 342-356.

[12] R. García and R. Gil, "Social Media Copyright Management using Semantic Web and Blockchain," in Proceedings of the 21st International Conference on Information Integration and Web-based Applications \& Services, 2019, pp. 339-343.

[13] E. Febriyanto, R. S. Naufal, and S. Sulistiawati, "Planning of the Web-based E-Raport Assessment System," Aptisi Trans. Technopreneursh., vol. 2, no. 1, pp. 48-58, 2020.

[14] S. Huang, G. Wang, Y. Yan, and X. Fang, "Blockchain-based data management for digital twin of product," J. Manuf. Syst., vol. 54, pp. 361-371, 2020.

[15] P. A. Sunarya, A. Khoirunisa, and P. Nursaputri, "Blockchain Family Deed Certificate for Privacy and Data Security," in 2020 Fifth International Conference on Informatics and Computing (ICIC), 2020, pp. 1-4.

[16] U. Rahardja, A. N. Hidayanto, T. Hariguna, and Q. Aini, "Design Framework on Tertiary Education System in Indonesia Using Blockchain Technology," 2019 7th Int. Conf. Cyber IT Serv. Manag. CITSM 2019, pp. 5-8, 2019, doi: 10.1109/CITSM47753.2019.8965380.

[17] I. Handayani, R. Supriati, and E. S. N. Aisyah, "Proof of Blockchain Work on The Security of Academic Certificates," in 2020 8th International Conference on Cyber and IT Service Management (CITSM), 2020, pp. 1-5.

[18] S. R. Niya, F. Maddaloni, T. Bocek, and B. Stiller, "Toward scalable blockchains with transaction aggregation," in Proceedings of the 35th Annual ACM Symposium on Applied Computing, 2020, pp. 308-315.

[19] U. Rahardja, E. P. Harahap, and D. D. Christianto, "PENGARUH TEKNOLOGI BLOCKCHAIN TERHADAP TINGKAT KEASLIAN IJAZAH,” Technomedia J., vol. 4, no. 2, pp. 211-222, 2020.

[20] L. Kristoufek, "Bitcoin and its mining on the equilibrium path," Energy Econ., vol. 85, p. 104588, 2020.

[21] D. Lee and N. Park, "Blockchain based privacy preserving multimedia intelligent video surveillance using secure Merkle tree," Multimed. Tools Appl., pp. 1-18, 2020.

[22] E. P. Harahap, Q. Aini, and R. K. Anam, "PEMANFAATAN TEKNOLOGI BLOCKCHAIN PADA PLATFORM CROWDFUNDING," Technomedia J., vol. 4, no. 2, pp. 199-210, 2020.

[23] B. L. Reynolds, "The effects of nonce words, frequency, contextual richness, and L2 vocabulary knowledge on the incidental acquisition of vocabulary through reading: more than a replication of Zahar et al.(2001) \& Tekmen and Daloğlu (2006)," Int. Rev. Appl. Linguist. Lang. Teach., vol. 58, no. 1, pp. 75-102, 2020.

[24] J.-F. Têtu, L.-C. Trudeau, M. Van Beirendonck, A. Balatsoukas-Stimming, and P. Giard, "A standalone FPGA-Based miner for Lyra2REv2 cryptocurrencies," IEEE Trans. Circuits Syst. I Regul. Pap., vol. 67, no. 4, pp. 1194-1206, 2020.

[25] U. Rahardja, T. Nurhaeni, A. Khoirunisa, and R. D. I'zzaty, "LTAI BERBASIS TEKNOLOGI BLOCKCHAIN UNTUK MENINGKATKAN ALEXA RANK," in SENSITIf: Seminar Nasional Sistem Informasi dan Teknologi Informasi, 2019, pp. 373-380.

[26] Z. Zheng, S. Xie, H. Dai, X. Chen, and H. Wang, "An overview of blockchain technology: Architecture, consensus, and future trends," in 2017 IEEE international congress on big data (BigData congress), 2017, pp. 557-564.

[27] G. Maulani, U. Rahardja, M. Hardini, R. D. l'zzaty, Q. Aini, and N. P. L. Santoso, "Educating Farmers Using Participatory Rural Appraisal Construct," in 2020 Fifth International Conference on Informatics and Computing (ICIC), 2020, pp. 1-8.

[28] H. Sanjuan, S. Poyhtari, P. Teixeira, and I. Psaras, "Merkle-CRDTs: Merkle-DAGs meet CRDTs," arXiv Prepr. arXiv2004.00107, 2020.

[29] H. Abbaspour, "Potential benefits of blockchain technology for mining industry: with a case study of truck dispatching system in open pit mines," 2018.

[30] M. Szydlo, "Merkle Tree Traversal in Log Space and Time (2003 Preprint version)."

[31] C. Stagnaro, "White paper: Innovative blockchain uses in health care," Free. Assoc., 2017.

[32] G. Nagasubramanian, R. K. Sakthivel, R. Patan, A. H. Gandomi, M. Sankayya, and B. Balusamy, "Securing e-health records using keyless signature infrastructure blockchain technology in the cloud," Neural Comput. Appl., vol. 32, no. 3, pp. 639-647, 2020.

[33] H. Huang, X. Chen, and J. Wang, "Blockchain-based multiple groups data sharing with anonymity and traceability," Sci. China Inf. Sci., vol. 63, no. 3, pp. 1-13, 2020.

[34] A. S. Bein, Y. I. Graha, and A. P. Pangestu, "Pandawan Website Design Based Content Management System As Media E-commerce Transaction," Aptisi Trans. 
Technopreneursh., vol. 2, no. 1, pp. 87-97, 2020.

[35] E. Guustaaf, U. Rahardja, Q. Aini, H. W. Maharani, and N. A. Santoso, "Blockchain-based Education Project," Aptisi Trans. Manag., vol. 5, no. 1, pp. 46-61, 2021.

[36] J. Vlieghe and P. Zamojski, "Education for Education's Sake: The Idea of a Thing-Centred Pedagogy," in Towards an Ontology of Teaching, Springer, 2019, pp. 11-28.

[37] P. A. Sunarya, Q. Aini, A. S. Bein, and P. Nursaputri, "The Implementation Of Viewboard Of The Head Of Department As A Media For Student Information Is Worth Doing Final Research," ITSDI J. Ed. Vol. 1 No. 1 Oct. 2019, p. 18, 2019.

[38] I. Noburu, A. Himki, A. Dithi, K. Kano, and M. Anggraeni, "Covid-19: Portrait of Preservation of the Batik Industry as a Regional Autonomy," Aptisi Trans. Technopreneursh., vol. 2, no. 2, pp. 143-152, 2020, doi: 10.34306/att.v2i2.91.

[39] U. R. Sudaryono, "Decision Support System for Ranking of Students in Learning Management System (LMS) Activities using Analytical Hierarchy Process (AHP) Method," in Journal of Physics: Conference Series, 2020, vol. 1477, p. 22022.

[40] Q. Aini, M. Yusup, N. P. L. Santoso, A. R. Ramdani, and U. Rahardja, "Digitalization Online Exam Cards in the Era of Disruption 5.0 using the DevOps Method," J. Educ. Sci. Technol., vol. 7, no. 1, pp. 67-75, 2021.

[41] P. A. Sunarya, N. Lutfiani, N. P. L. Santoso, and R. Ajeng, "The Importance of Technology to the View of the Qur'an for Studying Natural Sciences," Aptisi Trans. Technopreneursh., vol. 3, no. 1, pp. 58-67, 2021.

[42] A. Dudhat, N. P. L. Santoso, S. Santoso, and R. Setiawati, "Blockchain in Indonesia University: A Design Viewboard of Digital Technology Education," Aptisi Trans. Technopreneursh., vol. 3, no. 1, pp. 68-80, 2021.

[43] M. Kamil, A. S. Bist, U. Rahardja, N. P. L. Santoso, and M. Iqbal, "Covid-19: Implementation e-voting Blockchain Concept," Int. J. Artif. Intell. Res., vol. 5, no. 1, 2021. 